\title{
Acceso abierto a la producción científica de Uruguay: poca historia en 10 años (2009-2018)
}

Open access to Uruguayan scientific production: little history in 10 years (2009-2018)

\section{Natalia Aguirre-Ligüera}

Universidad de la República. Facultad de Información y Comunicación, Uruguay

natalia.aguirre@fic.edu.uy

Juan Maldini

Agencia Nacional de Investigación e Innovación, Uruguay

juanmaldini@gmail.com

\section{Exequiel Fontans}

Universidad de la República. Facultad de Información y Comunicación, Uruguay

exequiel.fontans@fic.edu.uy

\section{Resumen:}

Este trabajo analiza los avances del acceso abierto a la literatura científica en Uruguay en los últimos diez años. Se basa en un análisis cuantitativo de carácter exploratorio de los artículos publicados y en el relato de iniciativas locales. Se identifican políticas institucionales recientes y poco consolidadas, aún sin alcance nacional. Se analizan las publicaciones de autores con filiación nacional uruguaya en acceso abierto a partir de la WOS, encontrando un 37,3\% de artículos disponibles en forma inmediata y en constante aumento, empujado por la vía dorada. Se analizan las políticas editoriales de las revistas en Sherpa Romeo y se encuentra que el 50,7\% no está disponible en acceso abierto pero podría ser depositado en repositorios. Sumando ambas categorías se halla que un $88 \%$ de la producción considerada está o podría estar disponible en acceso abierto. Se concluye que es necesario generar estudios adicionales en torno al acceso abierto para Uruguay.

Palabras clave: Acceso Abierto, Uruguay.

\section{Abstract:}

This paper analyses the advances of open access to scientific literature in Uruguay during the last ten years. It is based on an exploratory quantitative study of published articles and a review of local initiatives. Institutional policies, which are not yet consolidated and have not reached a national level, are identified. The availability and evolution of open access to Uruguayan researchers' publications in the WOS is studied, finding 37,3\% of papers immediately available and consistent growth, based on the golden route. Editorial policies in Sherpa Romeo are analyzed for journals, finding 50.7\% of not open access articles that could be archived in institutional repositories. Both categories added show that $88 \%$ of considered production is or could be available in open access. The conclusions highlight a need to develop additional research around open access in Uruguay.

KeYwORDS: Open Access, Uruguay.

\section{INTRODUCCIÓN}

A 17 años de la primera declaración de acceso abierto de alcance global (Budapest Open Access Initiative, 2002) se impone una revisión sobre el impacto que ha tenido este movimiento que por lo menos desde principios de siglo, aunque con antecedentes en el siglo XX, propone el acceso sin barreras económicas, 
técnicas o de ningún otro tipo al conocimiento científico registrado a través de Internet, como alternativa a las conocidas contradicciones del modelo imperante basado en la publicación en revistas de suscripción.

El problema del acceso abierto al conocimiento científico registrado es un tema sensible en la comunidad internacional y particularmente en los países de la región latinoamericana, que cuentan con escasos recursos para la investigación, para el desarrollo de sistemas de información y para la adquisición de bibliografía especializada. La cuestión de que las instituciones de investigación pagan dos veces para la realización y acceso al conocimiento generado por sus propios investigadores, ha sido abordado ampliamente en la bibliografía producida a nivel mundial (Fecher y Friesike, 2014; Mittermaier, 2015) y específicamente en las reflexiones desde el Sur (Babini, 2014). Babini sostiene que:

En América Latina, tanto la investigación científica como la comunicación de sus resultados son actividades financiadas principalmente con fondos públicos; es decir que la participación estatal es protagonista en la actividad científica y en las comunicaciones científicas en la región (Babini, 2019).

En Uruguay la inversión pública en investigación significa un 70\% promedio del gasto total para el período 2009-2017, tanto para actividades de ciencia y tecnología en general como para innovación y desarrollo en particular (Agencia Nacional de Investigación e Innovación, 2019). En esta situación la problemática del acceso a la literatura científica involucra cuestionamientos vinculados a la transparencia en el uso de dineros públicos.

La influencia del movimiento de acceso abierto demoró en llegar a Uruguay. En 2009 la entonces Escuela Universitaria de Bibliotecología y Ciencias Afines, hoy Instituto de Información de la Facultad de Información y Comunicación, de la Universidad de la República (Udelar) toma el tema y presenta el proyecto $\mathrm{I}+\mathrm{D}$ Modelo de repositorio institucional como alternativa para el desarrollo social científico y tecnológico del país, a la convocatoria Proyectos I+D 2010 de la Comisión Sectorial de Investigación Científica (CSIC). El proyecto es financiado y se ejecuta entre 2010 y 2012. Su objetivo general era "contribuir al desarrollo social, científico y tecnológico del país a través de la promoción del libre acceso a la información y al conocimiento científico producido a nivel nacional” (Aguirre-Ligüera y Ceretta, 2014). Hasta ese momento no se encontraron antecedentes nacionales de abordajes académicos de la temática, aunque si se identificaron esfuerzos aislados y "voluntariosos" de bibliotecas universitarias o de investigación que impulsaban el desarrollo de repositorios, sin demasiados medios técnicos, ni apoyos institucionales. El proyecto finaliza en 2012 y presenta como resultados:

- Una caracterización de las publicaciones declaradas en el currículum vitae (CV) de los investigadores activos del Sistema Nacional de Investigadores (SNI) por áreas del conocimiento (Picco et al., 2014).

- Un análisis de la disponibilidad de la producción científica de las ciencias sociales nacionales a través del catálogo en línea de la Udelar (Fontans, Simón y Ceretta, 2015).

- Un análisis de las políticas editoriales de las revistas académicas resultantes a partir de las categorías utilizadas por Sherpa Romeo para determinar en qué medida esas contribuciones podrían estar disponibles en repositorios institucionales de acceso abierto.

- La constatación de la ausencia de políticas nacionales o institucionales en relación al acceso abierto al conocimiento en ciencia y tecnología, aunque se encontró preocupación y sensibilidad de las autoridades por generar mecanismos de democratización del acceso a los resultados de la investigación nacional.

- Dos trabajos en eventos internacionales sobre el estado de situación del acceso abierto en el país que presentan los principales resultados del proyecto (Ceretta y Aguirre, 2013; Aguirre y Ceretta, 2014).

Se considera a este proyecto como el puntapié inicial para el desarrollo del acceso abierto en Uruguay y principal antecedente para el presente trabajo. En primer lugar, porque contribuyó a instalar la discusión en 
ámbitos académicos e institucionales, y en segundo término porque generó los primeros datos encontrados sobre el acceso abierto a la producción nacional en ciencia y tecnología.

Este trabajo tiene como objetivo general describir el proceso de desarrollo del acceso abierto en Uruguay, en los últimos diez años. Para su consecución se plantean dos objetivos específicos: 1) describir las iniciativas de acceso abierto en Uruguay, en las vías verde y dorada; y, 2) analizar la disponibilidad en acceso abierto de la producción científica nacional del período, sus vías, su evolución y su potencial.

\section{Metodología}

El enfoque metodológico es de carácter descriptivo y exploratorio. La pregunta que motiva esta contribución, a 10 años del proyecto de investigación antes mencionado, es iqué avances es posible identificar en relación al acceso abierto a la literatura científica nacional?

Con el fin de dar respuesta a esta interrogante, el trabajo se lleva adelante de la siguiente manera:

Para alcanzar el objetivo 1 se realiza una revisión de las iniciativas nacionales de acceso abierto por vía verde y dorada, tomando como referencia algunos de los resultados arrojados por el proyecto mencionado para 2010-2012 y actualizándolos a 2019. Esta actualización se basa en fuentes secundarias, como ROARMAP, el sitio web de la Asociación Uruguaya de Revistas Académicas (AURA) (http://aura.edu.uy/), el repositorio de la Udelar Colibrí (https://www.colibri.udelar.edu.uy/jspui/), Latindex, SciELO y RedALyC; y a partir del conocimiento de los autores sobre los procesos abordados, para los que no se han encontrado fuentes primarias. Se construye, así un relato de carácter ensayístico que intenta reflejar el estado de situación a nivel nacional con respecto a las principales políticas e iniciativas de acceso abierto.

En Uruguay resulta muy difícil responder a la pregunta ¿cuál es la producción científica que realizan los investigadores nacionales? Los mecanismos de control bibliográfico que se ha dado el país funcionan, y parcialmente, para lo que se publica a nivel nacional, en especial para las monografías y en formato impreso. La legislación sobre depósito legal (Uruguay, 1970; 1971) no abarca a las publicaciones electrónicas y tampoco hay disposiciones que sancionen a las imprentas que no realicen el depósito. La producción nacional editada en el país en forma digital no es captada por la Biblioteca Nacional, mucho menos lo es aquello publicado en el exterior. Por lo tanto, las publicaciones de los investigadores uruguayos en revistas extranjeras resultan esquivas a los mecanismos de control bibliográfico. Hay algunos emprendimientos parciales como las bibliografías nacionales especializadas que generan anual o bianualmente las bibliotecas universitarias, como por ejemplo, la Biblioteca de Facultad de Ciencias de la Udelar, en la que se insta a los docentes a informar sobre sus publicaciones y se hacen búsquedas en bases de datos, con el fin de obtener un repertorio bibliográfico.

Una alternativa es extraer los datos de los CV de los investigadores nacionales, reunidos y categorizados en el Sistema Nacional de Investigadores. Se trata de datos auto-reportados por los investigadores, por lo que hay problemas en la consistencia y actualización de los mismos, que limitan su análisis (Aguirre-Ligüera et al., 2014).

En suma, no hay fuentes de datos nacionales que describan, brinden acceso a la producción científica nacional y posibiliten su estudio. Para hacerlo es necesario recurrir a bases de datos internacionales interdisciplinarias que posibilitan una mirada parcial sobre la producción nacional porque recogen revistas de corriente principal, dando lugar a sesgos idiomáticos, geográficos y disciplinares.

Para concretar el objetivo 2 se realiza un abordaje cuantitativo y descriptivo, utilizando dos fuentes de datos: la Web of Science (WOS) de la empresa Clarivate Analytics para obtener las publicaciones de autores nacionales en revistas de corriente principal, y Sherpa Romeo plataforma que mapea en forma colaborativa las políticas editoriales de las revistas a nivel mundial con respecto al depósito en repositorios de acceso abierto.

El universo de estudio está compuesto por el conjunto de las publicaciones (unidad de análisis) en las que participa al menos un autor con filiación institucional uruguaya, indizadas por la WOS en el período 
2009-2018. Se busca para esto en el campo Address de la Core Collection de WOS el término URUGUAY, estableciendo el período temporal 2009-2018 y se aplica el filtro Countries and Regions por Uruguay. Finalmente, se descartan aquellos registros que carecen de ISSN o de EISSN.

En tanto, la disponibilidad en acceso abierto de la producción científica nacional se analiza a partir de las categorías de análisis: acceso abierto real y potencial definidas por Miguel, Gómez y Bongiovani (2012). Estas autoras entienden que las publicaciones son de acceso abierto real cuando son "accesibles en abierto de manera inmediata o con algún período de embargo" y de acceso abierto potencial cuando "las revistas proveen los permisos para alguna forma de auto-archivo, y por tanto los textos completos podrían liberarse en repositorios o sitios web de los autores" (Miguel et al. 2012).

Para evaluar el acceso abierto real se toman las publicaciones categorizadas por la WOS en el campo OA y se analiza diacrónicamente de acuerdo con la vía por la que se concreta: dorada, verde y bronce. El campo OA en la WOS puede asumir los siguientes valores, no excluyentes, explicados en su sitio web (Clarivate Analytics, 2019):

- Dorado DOAJ para artículos publicados en revistas incluidas en el directorio DOAJ;

- Dorado Otro para artículos que, aunque no están en DOAJ tienen una licencia de Creative Commons (CC) y son levantados por la base de datos Unpaywall de Impactstory;

- Verde Publicado son versiones finales y publicadas de artículos que se alojan en repositorios de acceso abierto;

- Verde Aceptado son manuscritos aceptados alojados en repositorios, cuyo contenido pasó el peer review pero lo que está disponible no es la versión del editor;

- Bronce las licencias de estos trabajos no es clara o aparecen en Unpaywall como artículos sin licencia CC, se trata de artículos que pueden consultarse gratuitamente o se encuentran disponibles en los sitios web de los editores. La WOS advierte que los trabajos en esta última categoría pueden incluir contenido incompleto o haber sufrido modificaciones temporales, por ejemplo, porque una editorial para promocionarse pone un trabajo en su sitio en abierto por un período y luego lo cierra.

Se agrupan estas categorías en tres: Dorada (Dorado DOAJ y Dorado Otro), Verde (Verde Publicado y Verde Aceptado) y Bronce.

Se consulta Sherpa Romeo con el ISSN y EISSN y se clasifican las publicaciones de acuerdo con el color que les asigna. El Verde significa que permite el archivo del pre-print (versión anterior a la revisión), post-print (versión final posterior a la revisión) o la versión del editor/PDF. El Azul permite depositar el post-print o la versión del editor/PDF. El Amarillo permite depositar en un repositorio el pre-print. El Blanco indica que el archivo no está formalmente permitido (Sherpa Romeo, s.d.). Asimismo, la API arroja algunos títulos con el color Gris, que indica que no cuentan con datos para clasificar a esa revista. Para este estudio, se sumaron a la categoría Gris los títulos de revistas que no fueron encontrados en Sherpa Romeo.

El acceso potencial se estimó descartando aquellos registros caracterizados como de acceso abierto real, y considerando el color que le asigna a cada publicación Sherpa Romeo. Se realiza un análisis diacrónico de las publicaciones en función del color mencionado.

Se analiza la evolución del conjunto de publicaciones de acuerdo con su disponibilidad en acceso abierto: real, potencial, no admite acceso abierto y sin dato, cuatro categorías excluyentes. 


\section{Resultados y Discusión}

\subsection{Iniciativas nacionales de acceso abierto: vías verde y dorada}

En 2010 en el marco del proyecto mencionado se realizaron entrevistas a autoridades de instituciones clave para el sistema nacional de ciencia y tecnología, en las que se evidenciaba la preocupación por la democratización del conocimiento científico, en concordancia con el Plan Estratégico Nacional de Ciencia, Tecnología e Innovación (PENCTI) aprobado en 2010, cuyo objetivo tres consiste en: "Desarrollar capacidades y oportunidades para la apropiación social del conocimiento y la innovación inclusiva" (Uruguay. Gabinete Ministerial de la Innovación, 2010). No se mencionaban iniciativas de acceso abierto, sino la preocupación por generar políticas públicas que encuadren acciones específicas vinculadas al mismo. Todos los entrevistados manifestaban la necesidad de involucrar a los investigadores en los procesos de formulación y diseño de estas políticas, atendiendo a las particularidades de cada disciplina, considerando los mecanismos de evaluación, los contratos de los investigadores con organismos financiadores y la preocupación por no poner trabas a la publicación. Se encontraron opiniones divergentes respecto a quin debería diseñar e implementar eventuales políticas de acceso abierto y la posibilidad y conveniencia de generar mandatos en este sentido.

La principal estrategia para el acceso a la literatura científica fue el Portal Timbó (http://www.timbo.or g.uy) que ofrece una puerta de entrada gratuita a un importante conjunto de bases de datos de suscripción, entre otros recursos. El portal fue creado en 2009 por la Agencia Nacional de Investigación e Innovación y desde 2015 es accesible para cualquier persona desde una IP uruguaya.

Casi diez años después siguen sin existir políticas explícitas de acceso abierto de alcance nacional y los sistemas de evaluación de los investigadores siguen privilegiando la publicación en revistas de corriente principal. Caitán (2011, p.155) señalaba la necesidad de políticas públicas que contribuyeran a "cambiar el sistema de recompensas académico e invertir en sistemas emergentes de comunicación científica" y la oportunidad que significaba el acceso abierto. Aún no se cuenta con datos respecto a percepción y comportamientos de los académicos nacionales en relación al acceso abierto.

Una búsqueda en ROARMAP (s.d.), que recoge mandatos y políticas de autoarchivo de universidades, institutos de investigación y agencias financiadoras, no tiene ninguna entrada para Uruguay. A pesar de no estar reflejadas en esta base de datos, se conocen algunas políticas institucionales de acceso abierto que han sido fundamentales para la instalación del tema en ámbitos académicos. Entre ellas un avance fundamental hacia el acceso abierto a la producción académica nacional ha sido el proceso de implementación del Repositorio Colibrí de la Universidad de la República (Udelar), institución responsable de aproximadamente el 80\% del conocimiento registrado producido en el país (Aguirre-Ligüera y Ceretta, 2013; Bianco y Sutz, 2014; DICYT, 2012).

En 2012 se reúnen en Buenos Aires autoridades de ciencia y tecnología de varios países latinoamericanos con vistas a firmar el acuerdo marco para el lanzamiento de LA Referencia, Red Federada de Repositorios Institucionales de Publicaciones Científicas, que nace con nueve países socios. Participa de la reunión en representación de la Udelar la Dra. Ida Holz, quien a su regreso plantea la importancia de que Uruguay se sume a esta iniciativa regional. El país no adhiere al acuerdo de cooperación fundacional de LA Referencia, dado que no existían en ese entonces repositorios de acceso abierto ni proyectos concretos en esa dirección.

La Udelar toma el tema y a instancias de la CSIC reúne a un grupo de trabajo con vistas a generar una propuesta de repositorio institucional. Este grupo cuenta con la participación de autoridades universitarias, representantes de las bibliotecas universitarias, el Servicio Central de Informática Universitaria e investigadores de diferentes áreas del conocimiento. El repositorio se crea formalmente en 2014 y su política de acceso abierto se explicita en la ordenanza por la que se rige. Establece la obligatoriedad de depósito para todos sus docentes, y personas en relación de dependencia, sobre la producción relacionada con actividades de 
investigación y para los estudiantes en relación a las tesis y proyectos finales de carrera, entre otros contenidos (Universidad de la República, s.d.). Sin embargo, esta obligación solo se ve reflejada en los contenidos del repositorio para las tesis de posgrado y proyectos de grado.

A partir de esta iniciativa pionera otras universidades e institutos universitarios han generado sus propios repositorios institucionales.

A la fecha se conocen en producción el Repositorio Académico Digital de la Universidad ORT (http s://dspace.ort.edu.uy/), Ainfo del Instituto Nacional de Investigación Agropecuaria (http://www.ainfo.i nia.uy/consulta/), Colecciones Digitales de la Biblioteca Nacional -con algunas colecciones producto de sus actividades de investigación presentes en su repositorio de colecciones históricas digitalizadas- y los repositorios temáticos de la Fundación Ceibal (http://digital.fundacionceibal.edu.uy) y del Ministerio de Desarrollo Social (http://dspace.mides.gub.uy). De éstos solo el repositorio de la Fundación Ceibal explicita su política de acceso abierto. En distintos niveles de desarrollo se conocen también iniciativas de repositorios institucionales en la Universidad Católica del Uruguay, la Universidad de Montevideo, el Consejo de Formación Docente de la Administración Nacional de Educación Pública (CFE - ANEP), el Instituto Universitario Asociación Cristiana de Jóvenes (IUACJ) y la Agencia Nacional de Investigación e Innovación (ANII).

Estas iniciativas resultan un considerable avance en relación al relevamiento de sitios web de instituciones públicas que financian o ejecutan actividades de ciencia y tecnología en 2011. En ese momento no se identificaron sistemas de archivo y descripción de esa producción, que propiciaran su difusión y recuperación, por lo tanto, las publicaciones no tenían visibilidad ni dentro ni fuera de la institución. Además, se encontró que varias instituciones ni siquiera exigían a los investigadores el reporte de las publicaciones generadas, mientras que otras solicitaban la referencia y solo a veces se exigía la entrega del texto completo.

La ANII actualmente está en plena implementación de un proyecto cuyo principal objetivo es generar una red nacional de repositorios académicos de acceso abierto interoperables en ciencia y tecnología. A esos efectos ANII se incorporó recientemente como socio de LA Referencia constituyéndose en nodo nacional para Uruguay, que se convierte en el décimo país miembro de la red. Se espera que este proyecto contribuya al desarrollo de infraestructura, el diseño e implementación de políticas conjuntas que faciliten la interoperabilidad entre repositorios, la capacitación de recursos humanos y la promoción del acceso abierto entre investigadores y tomadores de decisiones.

En relación a la vía dorada en Uruguay, como en el resto de América Latina, hay una marcada tendencia a que las revistas académicas sean de acceso abierto, lo que se explicaría porque las universidades, institutos de investigación y otras organizaciones sin fines de lucro no han delegado la edición de revistas en editoriales comerciales. Cetto y otros sostienen que: "Una característica más de la región, que la distingue de otras regiones del mundo, es la noción de la información científicacomo un bien común y del consecuente acceso libre a esta información" (Cetto, Alonso-Gamboa, Packer, y Aguado-López,2015).

En consonancia, Chinchilla-Rodríguez, Miguel y Moya-Anegón (2012) en base a datos de Scopus encontraron que para América Latina y el Caribe el 73,9\% de las publicaciones son de acceso abierto, mientras que en Europa y América del Norte baja drásticamente a 6,9\% y 4,9\% respectivamente. La subrepresentación de revistas uruguayas en las bases de datos internacionales como Scopus no permiten comparar la situación de Uruguay en este marco.

La Asociación Uruguaya de Revistas Académicas (AURA), constituida oficialmente en 2015, reúne 45 revistas nacionales en actividad. Solamente 3 son publicadas por editoriales comerciales, el resto (93,3\%) se editan bajo la responsabilidad de instituciones académicas, asociaciones profesionales u organismos públicos y se disponen en abierto, en consonancia con la situación descrita para América Latina. Solo 3 títulos requieren pago para acceder al contenido, de los cuales uno se edita solo en papel.

AURA es la fuente más exhaustiva para analizar la situación actual de las revistas académicas en función del acceso abierto. Sin embargo, no se cuenta con datos históricos con los que poder contrastar, haciendo 
imposible cualquier análisis diacrónico. El registro de ISSN podría ser una fuente exhaustiva, pero no es posible filtrar publicaciones en actividad, ni diferenciar revistas académicas de otro tipo de publicaciones seriadas.

Para analizar el crecimiento de las revistas de acceso abierto o cerrado es necesario recurrir a fuentes internacionales que no brindan información histórica, pero para las cuales se cuenta con datos de 2013.

TABLA 1

Revistas nacionales según fuente de datos consultadas, frecuencia absoluta y tasa de crecimiento simple

\begin{tabular}{lccc}
\hline Fuente & $\mathbf{2 0 1 3}$ & $\mathbf{2 0 1 9}$ & $\begin{array}{c}\text { tasa crecimiento } \\
\text { simple }\end{array}$ \\
\hline DOAJ & 7 & 22 & $214 \%$ \\
SciELO & 10 & 25 & $150 \%$ \\
RedALyC & 2 & 9 & $350 \%$ \\
LATINDEX & 101 & 151 & $50 \%$ \\
\hline
\end{tabular}

Fuente: Elaboración propia.

La tabla 1 muestra el crecimiento que han tenido las revistas nacionales en distintas fuentes de datos consultadas. Los datos correspondientes al año 2013 fueron tomados de Ceretta y Aguirre-Ligüera (2013). Se aprecia un importante crecimiento en todas las fuentes en el número de revistas nacionales. Interesa el aumento de títulos en los portales DOAJ, SciELO y RedALyC que solo incluyen revistas arbitradas de acceso abierto, y en este periodo de 6 años se multiplicó por 3, por 2,5 y por 4,5 respectivamente. La presencia en estas fuentes de datos repercute fuertemente en la visibilidad de los trabajos de los investigadores uruguayos, sobre todo a nivel regional. Este aumento no necesariamente responde al surgimiento de nuevas revistas, sino también a la adopción de políticas de acceso abierto o la incorporación de criterios de calidad establecidos por los portales.

En Latindex el crecimiento es bastante más discreto. El número de títulos se multiplicó por 1,5 alcanzando una tasa de crecimiento del 50\%. Respecto a los títulos presentes en Latindex, como se dijo, se realizó la búsqueda por revistas en línea. De los 151 títulos recuperados, 34,4\% son revistas arbitradas y un 31,8\% son revistas de acceso abierto, la intersección de arbitradas y de acceso abierto representa un 27,1\%.

A partir de la diferencia entre las tasas de crecimiento para el período de las fuentes que sólo reúnen revistas de acceso abierto (con una media de 238\%) y Latindex (con 50\%) podría afirmarse que las revistas de acceso abierto han tenido un crecimiento mucho mayor que las de acceso cerrado. Sin embargo, la inclusión en ambos casos depende de criterios de calidad disímiles que impone cada fuente y de la voluntad del editor de registrarla. Por eso para demostrar esta presunción habría que hacer un relevamiento exhaustivo de las revistas académicas existentes a nivel nacional, sus políticas de acceso y su evolución.

\subsection{Disponibilidad en acceso abierto de la producción científica nacional}

\section{Acceso abierto real}

La consulta en WOS arroja 15056 registros con al menos un autor con filiación uruguaya para el período 2009-2018. Se descartaron los que no tenían ISSN ni EISSN, resultando 14447.

De este universo y según la misma fuente 5389 (37.3\%) están disponibles en acceso abierto real a la fecha, distribuidos en:

- Vía dorada: $3993(74,1 \%)$ 
- Vía verde: $1641(30,4 \%)$

- Bronce: $1087(20,2 \%)$

Cabe aclarar que estas categorías no son mutuamente excluyentes por lo cual un artículo puede estar disponible en acceso abierto por más de una vía.

Minniti Santoro y Belli (2018) calculan el porcentaje de documentos en acceso abierto en 23,7\% para el Cono Sur (Argentina, Brasil, Chile, Paraguay y Uruguay) para el período 2005-2017, muy por encima de otras regiones latinoamericanas. Los autores observan la evolución histórica de los datos para ese período encontrando que oscila entre el $12 \%$ y el $28 \%$. Para Uruguay encuentran un porcentaje entre $11 \%$ y $13 \%$ de publicaciones en acceso abierto. Llama la atención la diferencia entre estos datos y los hallados en el presente trabajo para Uruguay en la misma fuente (37.3\%), muy por encima del valor para el Cono Sur (23,7\%), que incluye países con políticas fuertes de acceso abierto. Si se toma solo el período que comparten ambos trabajos (2009-2016) se encuentra que Uruguay se mantiene siempre por encima del 30\%, con valores muy cercanos al $40 \%$ para los últimos 4 años, mientras que el estudio citado encuentra una proporción que oscila entre el 24 y el 28\% para el Cono Sur. Se toman con cautela los datos de la categoría bronce ya que no hay certeza sobre la permanencia en acceso abierto de las publicaciones clasificadas como tal. Pero incluso si se consideraran las que solo son bronce como publicaciones de acceso cerrado, la disponibilidad hallada para Uruguay está muy por encima de los datos de la región.

Probablemente la diferencia se deba a la introducción por parte de la WOS de nuevas herramientas para identificar publicaciones en acceso abierto y clasificarlas. Pequeñas diferencias en las bases de datos incluidas en la búsqueda en WOS pueden contribuir también a explicar el dato llamativamente alto para Uruguay.

Volviendo al período de este trabajo se observa un crecimiento sostenido del porcentaje de publicaciones en acceso abierto. Esto va en consonancia con la tendencia a nivel mundial donde la prevalencia de los artículos de revistas académicas en acceso abierto parece ir en franco aumento. Piwowar et al. (2018) en un amplio estudio hallaron un crecimiento significativo y sostenido de la misma en relación al total de artículos con DOI en Crossreff, por lo que sostienen que "more recent articles are more likely to be OA, with the most recent year examined also containing the most OAs".

La figura 1 ilustra la evolución en la disponibilidad de las vías del acceso abierto real: verde, bronce y dorada para Uruguay.

\section{FIGURA 1}

Porcentaje de publicaciones uruguayas en acceso abierto real, según vía

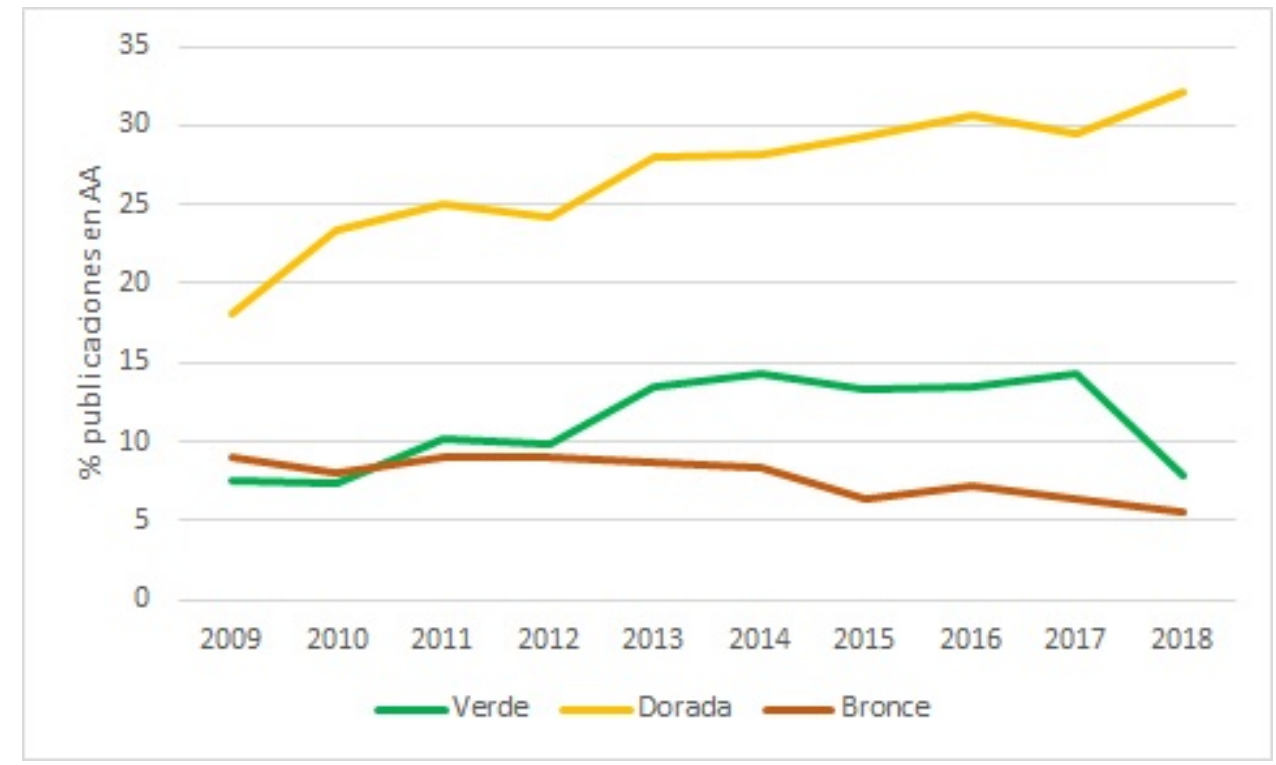


Llama la atención la meseta que presenta la vía verde, más o menos estable a partir de 2013. En tanto que el marcado descenso para 2018 se explica por los períodos de embargo impuestos por muchos editores para el depósito. Se asume que los repositorios donde está disponible esta producción no son repositorios institucionales uruguayos sino de instituciones de coautores extranjeros o repositorios temáticos, ya que no se conocen repositorios institucionales nacionales que permitan la cosecha de metadatos por OAI-PMH a la fecha.

La vía dorada es la que sostiene el crecimiento del acceso abierto real en Uruguay para el período. Sería muy interesante identificar cuánto de este crecimiento descansa sobre el cobro de tasas de procesamiento de artículos (APC), práctica habitual que muchos editores han adoptado como forma de adecuar sus modelos de negocio.

Los datos de que se dispone no son suficientes para analizar si el pago de APC afecta el acceso abierto a nivel nacional, porque tanto la categoría Dorado DOAJ como Dorado Otro pueden incluir publicaciones en estas condiciones. En DOAJ, según Abadal (2017), el 20\% de las revistas cobraba APC, el 45\% no lo hacía y no había datos para el resto. En relación a los artículos de categoría Dorado Otro mayormente provienen de revistas híbridas, como lo expresa la propia WOS. En este sentido Laakso y Björk (2016) encuentran que de 2007 a 2013 la cantidad de artículos de acceso abierto en revistas de suscripción se incrementó en más de veinte veces, considerando los principales editores comerciales. A partir de estos datos podría presumirse que el pago de APC podría incidir en el crecimiento de la vía dorada y por lo tanto del acceso abierto real a nivel nacional.

De constatarse el pago de APC por parte de los investigadores uruguayos y no habiendo políticas nacionales que los obliguen o estimulen a hacer disponible en abierto su producción, ¿qué podría estar motivándolos a pagar APC? Una posible respuesta sería la ventaja de visibilidad e impacto que tienen los trabajos en acceso abierto, cuidadosamente abordada por Hitchcock (2013) que generó una bibliografía sobre el tema a partir de los primeros estudios y la ha ido actualizando. Otra posible respuesta es que, en la Udelar -principal productora de literatura científica del país- está muy arraigada la idea del conocimiento científico como bien común. Los investigadores podrían estar pagando APC por una genuina preocupación de volver disponibles los resultados de su trabajo.

También es posible que los autores no estén pagando APC y la presencia de artículos con categoría Dorado Otro se explique porque las propias revistas de suscripción los liberan en acceso abierto, con fines promocionales o pasado un período de embargo, mientras que los artículos en Dorado DOAJ serían en revistas de acceso abierto que no cobran APC.

\section{Acceso abierto potencial}

Al fin de comparar la evolución del acceso abierto potencial desde la investigación tomada como antecedente de este trabajo hasta la actualidad, se consulta Sherpa Romeo para determinar en qué medida la producción uruguaya de 2009 a 2018 indizada en la WOS, podría ser depositada en un repositorio de acceso abierto. Se encuentra que al menos un $72 \%$ de la producción analizada puede depositarse, dato consistente, aunque levemente inferior, al reportado por Laakso (2013) para una amplia muestra de artículos de Scopus a nivel global. En tanto para 2009-2010 el 51\% de artículos de investigadores uruguayos, según su CV, permitía el depósito de alguna versión, sobre un total de 2313 (Aguirre-Ligüera y Ceretta, 2014). La figura 2 muestra la producción relevada en ambos trabajos según la clasificación de Sherpa Romeo, se observa que el porcentaje de lo que no admite formalmente depósito varía en un punto porcentual, y que la mayor distancia se establece entre los trabajos en la categoría gris, sin dato. Los resultados actuales son mucho más favorables al acceso abierto, que los precedentes, pero no tanto en 
detrimento de las publicaciones que no permiten archivo (blanco) sino de aquellas que no presentan datos.

FIGURA 2

Distribución del conjunto de las publicaciones (CVUy y WOS), según clasificación de Sherpa Romeo

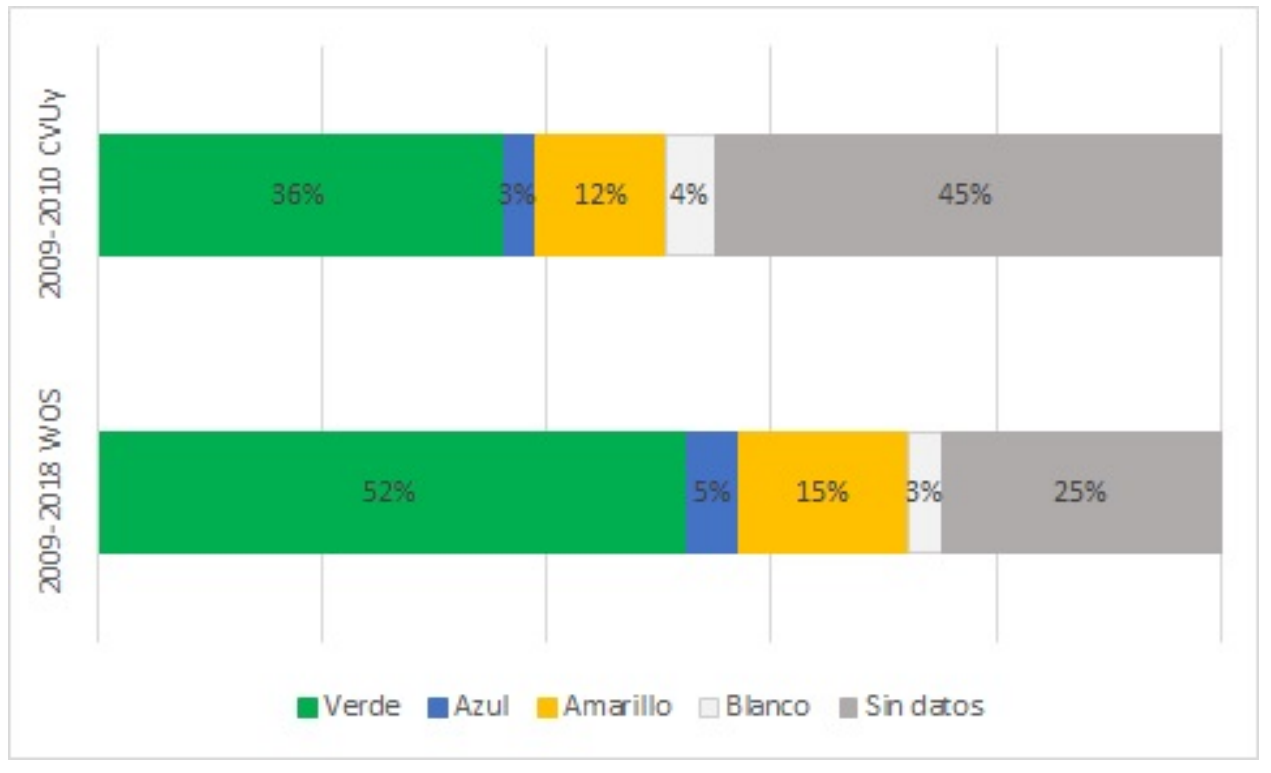

Fuentes: CVUy, WOS, Sherpa Romeo. Elaboración propia.

La comparación entre ambos estudios presentada en la figura 2 tiene sus limitaciones por tratarse de fuentes de datos distintas. La WOS reúne revistas de corriente principal, mejor representadas en Sherpa Romeo que las reportadas en el CV por investigadores nacionales. Entre las últimas posiblemente se encuentre un importante número de títulos nacionales o de escasa visibilidad internacional, con diferencias por área del conocimiento (Picco et al. 2014). Otra limitación para la comparación son los períodos considerados: uno de dos años incluido en el otro de diez años. Sin embargo, acotando las publicaciones tomadas de WOS a 2009-2010 se encuentran resultados no demasiado dispares a los de la década.

Volviendo a centrar el foco en los resultados de este trabajo, en el mismo se consideran las categorías de acceso abierto real y potencial como excluyentes, por lo tanto, una vez determinado el acceso abierto real, como se explicó en el apartado anterior, se identifica el acceso abierto potencial a las publicaciones restantes, que ascienden a 9058. Al desagregar por color asignado en Sherpa Romeo se aprecia que la categoría verde es ampliamente mayoritaria, representando 59,2\%, seguida por amarilla y azul con $18,6 \%$ y $3,1 \%$ respectivamente. De este grupo de publicaciones las que no permiten depósito, color blanco, significan el $4 \%$. No se obtuvieron datos del 15,1\% ya sea porque no figuran en Sherpa o porque las clasifica como gris. 
FIGURA 3

Evolución diacrónica de las publicaciones (excluyendo las de acceso abierto real) indizadas en la WOS, según categoría de Sherpa Romeo

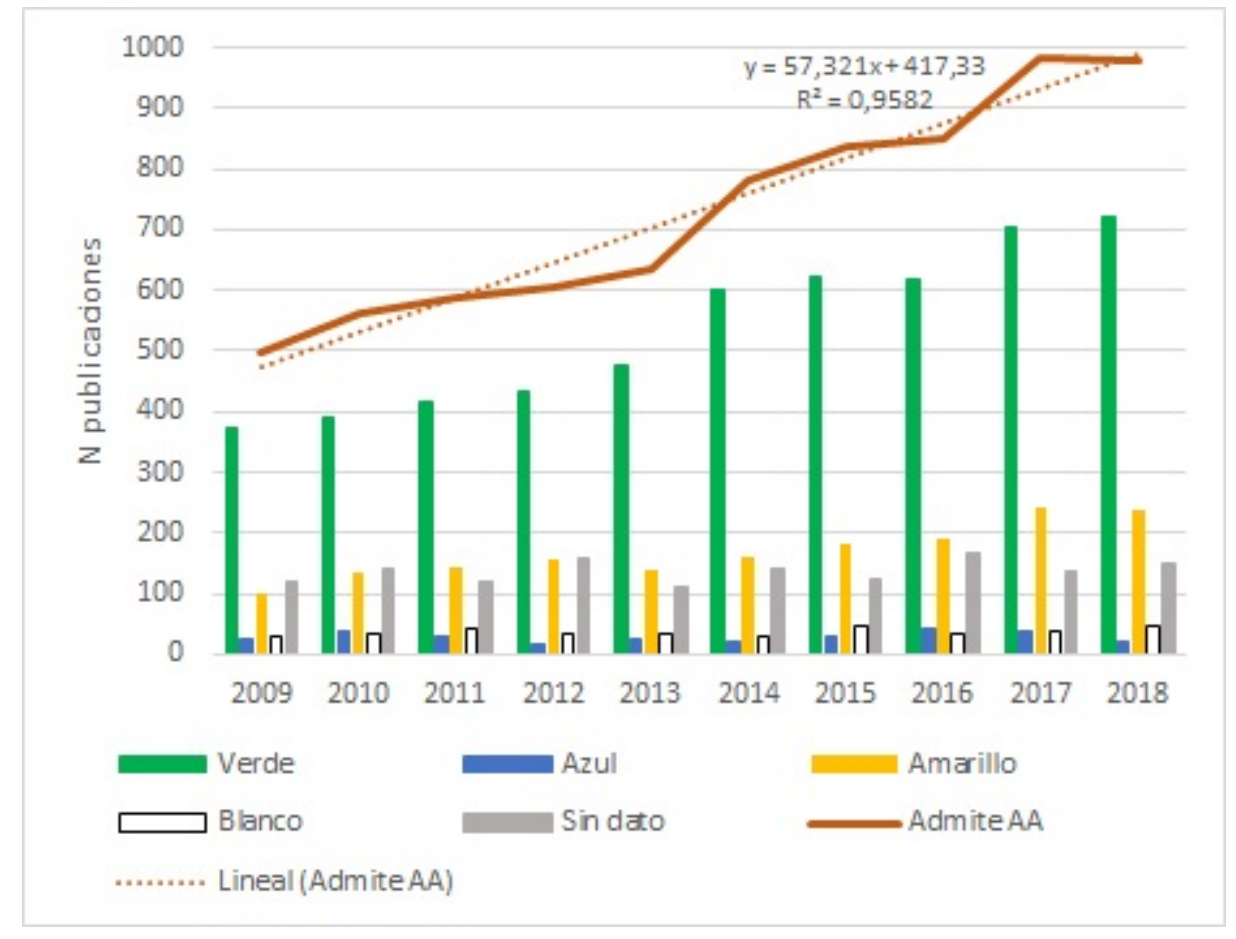

Fuentes: WOS y Sherpa Romeo. Elaboración propia.

La figura 3 muestra una marcada tendencia al alza para las categorías verde, amarilla y las publicaciones sin datos, el comportamiento de las categorías azul y blanco es más errático. La figura también presenta una línea que indica la tendencia creciente de la suma de las tres categorías que permiten algún tipo de depósito: verde, azul y amarillo.

En relación con el total de las publicaciones del período, 14447, las que no están disponibles en acceso abierto, pero podrían estarlo a través del depósito de alguna versión en repositorios ascienden a 50,7 \%. Si a esto se suma el 37,3\% hallado en acceso abierto real para el período, se encuentra que el $88 \%$ de la producción considerada está o podría estar disponible en acceso abierto sin afectar derechos de autores y editores. Solo para el 2,5\% no está formalmente admitido el depósito en repositorios. El restante 9,5\% no está en acceso abierto real y se desconoce si podría ser depositado en repositorios, por no tener datos al respecto. 
FIGURA 4

Evolución diacrónica de las publicaciones según acceso abierto, porcentaje

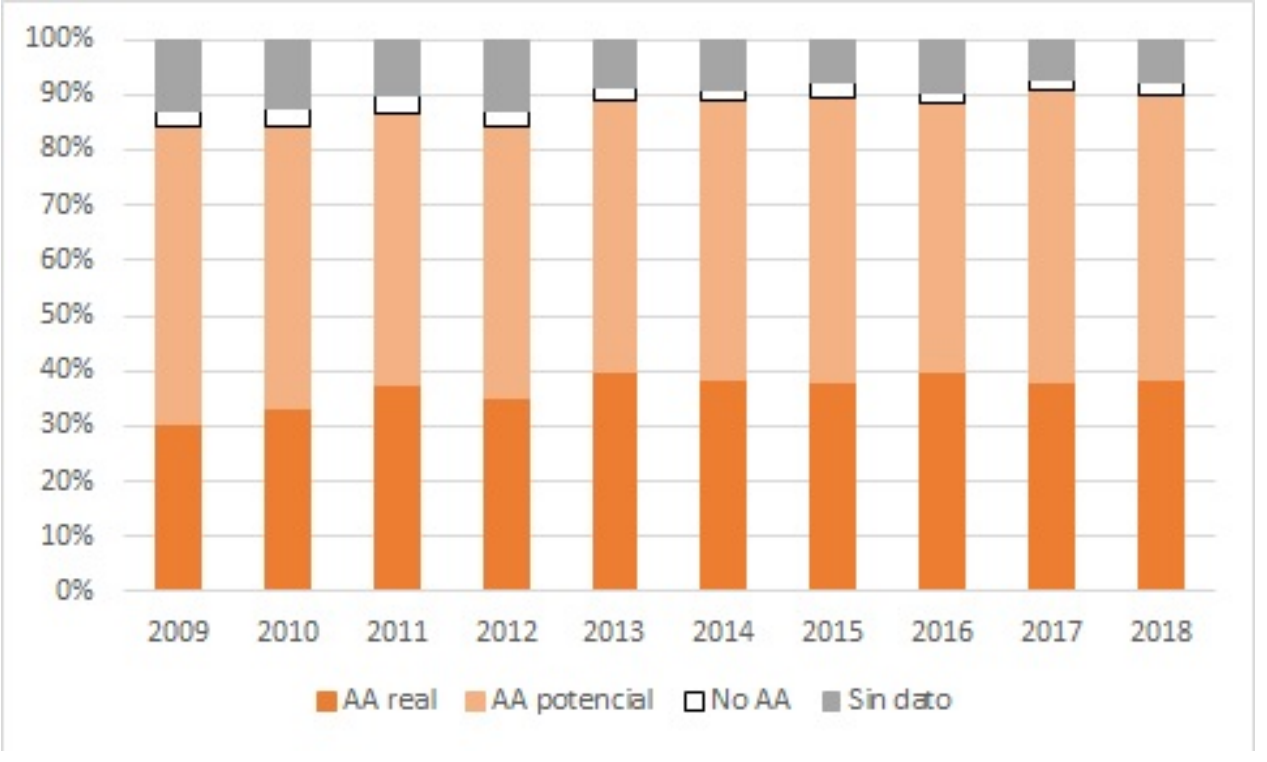

Fuentes: WOS y Sherpa Romeo. Elaboración propia.

La figura 4 ilustra la evolución de estos valores para el período considerado, mostrando una leve tendencia al alza de la suma de las publicaciones efectivamente disponibles o potencialmente disponibles en acceso abierto, con un promedio de $86 \%$ para el primer quinquenio considerado y de $90 \%$ para el segundo.

Miguel et al. (2012) hallaron para la producción argentina recogida en Scopus, para el período 2008-2010, que el $25,5 \%$ era de acceso abierto real, 44,2\% de acceso abierto potencial y 30,3\% sin información. Las autoras establecieron que el 69,7\% de los trabajos argentinos estaban o podrían estar en acceso abierto. Más allá de las diferencias entre el estudio mencionado y el presente, en las fuentes, los períodos y la forma de medir la categoría acceso abierto real, se puede afirmar que el escenario uruguayo actual es bastante más favorable al acceso abierto.

\section{CONSIDERACIONES FINALES}

Se identificaron algunas iniciativas que podrían generar cambios sustantivos en el acceso a la literatura científica nacional por vía verde en Uruguay, aunque todavía la situación de los repositorios académicos nacionales es incipiente y requiere de políticas de depósito claras y efectivas, fortalecimiento de infraestructura, políticas de interoperabilidad, coordinación interinstitucional, entre otros.

Las revistas académicas que se detectaron a nivel nacional son mayoritariamente de acceso abierto y han ganado visibilidad en portales regionales e internacionales en el período. Esto se explicaría porque están trabajando asociadas en la adopción de criterios de calidad.

A 10 años del estudio que sirve como antecedente se encuentran las mismas dificultades para identificar la producción científica nacional.

El 88\% de la producción nacional del período está o podría estar disponible en acceso abierto, sumando los resultados de acceso abierto real y potencial, se observa un crecimiento sostenido en ambas categorías.

El acceso abierto real a los artículos de autores nacionales representa un 37,3\%, porcentaje excepcionalmente alto en relación a lo reportado en estudios para la región. Este aumento se sostiene sobre la vía dorada. No hay indicios de que responda a cambios en la valoración de los investigadores con relación a la publicación o depósito en acceso abierto, ni estudios al respecto. Es necesario analizar la incidencia del pago de APC. En nuestra opinión el problema del acceso a la literatura científica no puede solucionarse con 
un cambio de modelo pagar por acceder a pagar por publicar, menos aún en países como Uruguay donde en ambos casos los fondos serían en su mayor parte públicos.

El acceso abierto potencial a los artículos de autores uruguayos que no están en acceso abierto real tiende al alza y representa el 50,7\% del total de publicaciones. Esto sustenta la idea de que una estrategia nacional centrada en repositorios sigue siendo viable.

Para finalizar, se debe destacar que no se identifican investigaciones vinculadas al tema en Uruguay desde 2014. Resulta entonces necesario el desarrollo de estudios adicionales sobre la producción científica nacional, su disponibilidad en acceso abierto, los factores que inciden en la misma, opiniones y hábitos de los investigadores nacionales, entre otros. De esta forma se podrá situar al país en el contexto regional e internacional y evaluar el impacto de las incipientes políticas identificadas.

\section{REFERENCIAS}

Abadal, E. (2017). Las revistas científicas en el contexto del acceso abierto. En E. Abadal (Ed.), Revistas cientificas: situación actual y retos de futuro (pp. 181-195). Barcelona: Universitat de Barcelona. Recuperado de http://eprints.rclis.org/32137/1/Revistas\%20cientificas\%202017\%209\%20Abadal\%20Ernest\%20Context o\%20del\%20acceso\%20abierto\%20p\%20181-195.pdf

Aguirre-Ligüera, N., y Ceretta, M.G. (2014). Construyendo un modelo de repositorio de acceso abierto para Uruguay. En Globalizaçao, Ciencia, Informaçao: atas VI Encuentro Ibérico EDICIC (pp. 1209-1219). Oporto, Portugal: CETAC MEDIA. Recuperado de http://www.youblisher.com/p/745142-VI-Encontro-Iberico-EDICIC-201 3-Globalizacao-Ciencia-Informacao/

Aguirre-Ligüera, N., Fontans, E., y Simón, L. (2014). El currículum vitae como fuente de datos en los estudios métricos. En Actas III Jornadas de Intercambio y Reflexión acerca de la Investigación en Bibliotecología, La Plata, Argentina. Recuperado de http://www.memoria.fahce.unlp.edu.ar/trab_eventos/ev.3077/ev.3077.pdf

Agencia Nacional de Investigación e Innovación (2019). Portal prisma [base de datos]. Recuperado de ANII https:/ /bi.anii.org.uy/eportal/group/anii-prisma

Babini, D. (2019). La comunicación científica en América Latina es abierta, colaborativa y no comercial. Desafíos para las revistas. Palabra Clave (La Plata), 8(2), e065. https://doi.org/10.24215/18539912e065

Babini, D. (2014, 21 de octubre). Universidades y acceso abierto: hora de tomar protagonismo. Foro Revista Iberoamericana de Ciencia, Tecnología y Sociedad. Recuperado de http://www.revistacts.net/elforo/627-el-deba te-universidades-y-acceso-abierto-es-hora-de-convertirnos-enprotagonista

Bianco, M., y Sutz, J. (2014). Introducción: sobre lo que trata este libro y sobre quién lo produjo. En Autoras (Eds.), Veinte años de politicas de investigación en la Universidad de la República: aciertos, dudas y aprendizajes. Montevideo: Udelar. CSIC. Recuperado de https://www.colibri.udelar.edu.uy/jspui/bitstream/20.500.12008/4149/1/Libro_Veinte_a\%C3\%B1os_de _pol\%C3\%ADticas_de_investigaci\%C3\%B3n_en_la_Universidad_de_la_Rep\%C3\%BAblica.pdf

Budapest Open Access Initiative. (2002). Recuperado de http://www.budapestopenaccessinitiative.org/read

Caitán, N. (2011). Acceso al conocimiento científico-tecnológico: el rol de las políticas públicas. e-colabora: revista de ciencia, educación, innovación y cultura apoyadas por redes de tecnología avanzada, 1(2), 146-162. Recuperado de http://eprints.rclis.org/16157/1/AccesoCCTPol\%C3\%ADticas_ecolabora.pdf

Ceretta, M.G., y Aguirre-Ligüera, N. (2013). Movimiento de Acceso Abierto en Uruguay: estado de situación y proyección. Trabajo presentado en Acceso Abierto, preservación digitaly datos cientificos III Conferencia Bibliotecas y Repositorios Digitales de América Latina BIREDIAL '13. San José de Costa Rica: Universidad de Costa Rica. Recuperado de https://biredial.ucr.ac.cr/index.php/Biredial2013/ai/paper/viewFile/18/40

Cetto, A.M, Alonso-Gamboa, J. O., Packer, A.L., y Aguado-López, E. (2015). Enfoque regional a la comunicación científica. Sistemas de revistas en acceso abierto. En J.P. Alperín y G. Fischman (eds.). Hecho en Latinoamérica: acceso abierto, revistas académicas e innovaciones regionales (pp. 19-41). Buenos Aires: CLACSO. 
Chinchilla-Rodríguez, Z., Miguel, S., y Moya-Anegón, F. (2012). Influencia del acceso abierto en las revistas de América Latina en el contexto internacional de la ciencia. e-colabora: revista de ciencia, educación, innovación y cultura apoyadas por redes de tecnología avanzada, 2(4), 28-48. Recuperado de http://www.memoria.fahce.unlp.edu.ar /art_revistas/pr.7262/pr.7262.pdf

Clarivate Analytics (2019). Colección principal de Web Of Science Ayuda. Recuperado de https://images.webofknowl edge.com//WOKRS532MR24/help/es_LA/WOS/hp_results.html\#dsy10670-TRS_open_access

DICYT (2012). Informe a la sociedad: ciencia, tecnología e innovación en Uruguay en los últimos años. Montevideo: DICYT. Recuperado de Ministerio de Educación y Cultura de https://www.mec.gub.uy/innovaportal/file/21 653/1/ciencia-tecnologia-innovacion-uruguay-ultimos-anios.pdf

Fecher, B. y Friesike, S. (2014). Open Science: One Term, Five Schools of Thought. En S. Bartling, y S. Friesike (Eds.), Opening Science: The Evolving Guide on How the Internet is Changing Research, Collaboration and Scholarly Publishing / (pp. 17-47). Cham: Springer.

Fontans, E., Simón, L. y Ceretta, M. G. (2015). Acceso a la producción científica financiada con fondos públicos: el caso de los investigadores del Área Social del SNI de Uruguay. Interciencia, 40(9), 588-595. Recuperado de http s://www.interciencia.net/wp-content/uploads/2017/10/588-FONTANS-8.pdf

Hitchcock, S. (2013). The effect of open access and downloads ('bits') on citation impact: a bibliography of studies. Recuperado de https://eprints.soton.ac.uk/354006/1/oacitation-biblio-snapshot0613.html

Laakso, M., y Björk, B.-C. (2016). Hybrid open access. A longitudinal study. Journal of Informetrics, 10(4), 919-932. https://doi.org/10.1016/j.joi.2016.08.002

Laakso, M. (2013). Green open access policies of scholarly journal publishers: a study of what, when, and where selfarchiving is allowed. Scientometrics, 99(2), 475-494. https://doi.org/10.1007/s11192-013-1205-3

Miguel, S., Gómez, N.D., y Bongiovani, P. (2012). Acceso abierto real y potencial a la producción científica de un país. El caso argentino. El profesional de la información, 21(2), 146-153. http://dx.doi.org/10.3145/epi.2012.mar.04

Minniti, S., Santoro, V., y Belli, S., (2018). Mapping the development of Open Access in Latin America and Caribbean countries. An analysis of Web of Science Core Collection and SciELO Citation Index (2005-2017). Scientometrics, 117(3), 1905-1930. https://doi.org/10.1007/s11192-018-2950-0

Mittermaier, B. (2015). Double Dipping in Hybrid Open Access - Chimera or Reality? ScienceOpen Research, 63(8). https://doi.org/10.14293/S2199-1006.1.SOR-SOCSCI.AOWNTU.v1

Picco, P., Aguirre-Ligüera, N., Maldini, J., Simón, L., Petroccelli, P., Fontans, E., Fager, J., y Ceretta, M. G. (2014). La comunicación científica en Uruguay: estudio de las publicaciones de los investigadores activos del Sistema Nacional de Investigadores (2009-2010). Transinformação, 26(2), 155-165. https://dx.doi.org/10.1590/0103 $-37862014000200005$

Piwowar, H., Priem, J., Larivière V., Alperin J.P., Matthias L., Norlander B., Farley A., West J., y Haustein S. (2018). The state of OA: a large-scale analysis of the prevalence and impact of Open Access articles. PeerJ, 6, e4375. h ttps://doi.org/10.7717/peerj. 4375

ROARMAP (s.d.). Registry of Open Access Repository Mandates and Policies. Recuperado de https:// roarmap.eprints.org/

Sherpa Romeo (s.d.). Search - Publisher copyright policies \& self-archiving. Recuperado de http://sherpa.ac.uk/romeo /search.php?la=en\&flDnum $=\mid \&$ mode $=$ simple

Universidad de la República (s.d.). Ordenanza del Repositorio Abierto de la Universidad de la República. Recuperado de https://www.colibri.udelar.edu.uy/jspui/Documentos/Ordenanza-aprobada.pdf

Uruguay. Gabinete Ministerial de la Innovación. (2010). Plan Estratégico Nacional de Ciencia Tecnología e Innovación. Recuperado de Agencia Nacional de Investigación e Innovación: http://www.anii.org.uy/institucional/docum entos-de-interes/23/plan-estrategico-nacional-de-ciencia-tecnologia-e-innovacion-pencti/

Uruguay (1970). Ley No13.835 Rendición de cuentas y balance de ejecución presupuestal. Ejercicio 1968. (art.191 y 193). Recuperado de https://www.impo.com.uy/bases/leyes/13835-1970

Uruguay (1971). Decreto No694/971 Derecho de autor. Depósito Legal. Recuperado de https://www.impo.com.uy/b ases/decretos/694-1971/7 